\title{
An In Vivo, MRI-Integrated Real-Time Model of Active Contrast Extravasation in Acute Intracerebral Hemorrhage
}

\author{
R.I. Aviv, T. Huynh, Y. Huang, D. Ramsay, P. Van Slyke, D. Dumont, P. Asmah, R. Alkins, R. Liu, and K. Hynynen
}

\begin{abstract}
BACKGROUND AND PURPOSE: The "spot sign" or contrast extravasation is strongly associated with hematoma formation and growth. An animal model of contrast extravasation is important to test existing and novel therapeutic interventions to inform present and future clinical studies. The purpose of this study was to create an animal model of contrast extravasation in acute intracerebral hemorrhage.
\end{abstract}

MATERIALS AND METHODS: Twenty-eight hemispheres of Yorkshire male swine were insonated with an MR imaging-guided focused sonography system following lipid microsphere infusion and mean arterial pressure elevation. The rate of contrast leakage was quantified by using dynamic contrast-enhanced MR imaging and was classified as contrast extravasation or postcontrast leakage by using postcontrast T1. Hematoma volume was measured on gradient recalled-echo MR imaging performed 2 hours postprocedure. Following this procedure, sacrificed brain was subjected to histopathologic examination. Power level, burst length, and blood pressure elevation were correlated with leakage rate, hematoma size, and vessel abnormality extent.

RESULTS: Median (intracerebral hemorrhage) contrast extravasation leakage was higher than postcontrast leakage (11.3; 6.3-23.2 versus 2.4; $1.1-3.1 \mathrm{~mL} / \mathrm{min} / 100 \mathrm{~g} ; P<.001)$. Increasing burst length, gradient recalled-echo hematoma $(\rho=0.54 ; 95 \% \mathrm{Cl}, 0.2-0.8 ; P=.007)$, and permeability were correlated $(\rho=0.55 ; 95 \% \mathrm{Cl}, 0.1-0.8 ; P=.02)$. Median permeability $(P=.02)$, gradient recalled-echo hematoma $(P=.02)$, and dynamic contrast-enhanced volumes $(P=.02)$ were greater at $1000 \mathrm{~ms}$ than at $10 \mathrm{~ms}$. Within each burst-length subgroup, incremental contrast leakage was seen with mean arterial pressure elevation $(\rho=0.2-0.8)$.

CONCLUSIONS: We describe a novel MR imaging-integrated real-time swine intracerebral hemorrhage model of acute hematoma growth and contrast extravasation.

ABBREVIATIONS: $\mathrm{CE}=$ contrast extravasation; $\mathrm{DCE}=$ dynamic contrast-enhanced; GRE = gradient recalled-echo; $\mathrm{ICH}=$ intracerebral hemorrhage; IQR $=$ interquartile range; $\mathrm{KPS}=$ rate of leakage; $\mathrm{MAP}=$ mean arterial pressure elevation; $\mathrm{PCL}=$ postcontrast leakage

ntracerebral hemorrhage (ICH) accounts for $10 \%-30 \%$ of strokes and is the most deadly and disabling stroke type with little improvement in mortality seen during the past 20 years. ${ }^{1}$ These characteristics underscore the importance of developing a

Received October 3, 2013; accepted after revision February 2, 2014.

From the Department of Medical Imaging (T.H., R.I.A., R.L.), Sunnybrook Health Sciences Centre, Toronto, Ontario, Canada; Departments of Medical Biophysics (K.H D.D., R.A., P.A.) and Medical Imaging (T.H., K.H., R.I.A.), University of Toronto, Toronto, Ontario, Canada; Department of Pathology (D.R.), London Health Sciences Centre, London, Ontario, Canada; and The Centre for Proteomic Studies (D.D., P.V.S.) and Imaging Research (Y.H., K.H.), Sunnybrook Research Institute, Toronto, Ontario, Canada.

Dr Aviv was supported by a grant from the Heart and Stroke Foundation of Canada (grant No. 7299). Dr Huynh was supported by grants from Canadian Institute for Health Research and the Physician Services Incorporated Foundation.

Please address correspondence to Richard I Aviv, MD, Sunnybrook Health Sciences Centre, 2075 Bayview Ave, Room AG-31E, Toronto, ON, M4N 3M5 Canada; e-mail: Richard.aviv@sunnybrook.ca

- Indicates open access to non-subscribers at www.ajnr.org

http://dx.doi.org/10.3174/ajnr.A3939 better understanding of the pathophysiology of ICH formation and growth to facilitate the development of improved therapeutic agents or interventions. ${ }^{2}$ The causative lesion in primary ICH is yet to be elucidated, though pathologic studies demonstrate focal vessel integrity loss in association with blood extravasation into the brain parenchyma. ${ }^{3}$ Following initial ICH formation, continuous $^{4,5}$ or delayed ${ }^{6}$ extravasation results in hematoma expansion, ${ }^{7}$ which is associated with early neurologic deterioration and significant mortality. ${ }^{8}$

Several recent studies have shown an association between contrast extravasation (CE) detected on CTA, coined the CTA "spot sign," and hematoma growth. ${ }^{9-14}$ Prospective studies have demonstrated that contrast extravasation independently predicts a larger hematoma size and a poorer clinical outcome. ${ }^{13,14}$ These are the first clinical studies to suggest a robust "real-time" imaging marker of hematoma expansion. Three clinical studies are presently enrolling patients dichotomized by the CTA spot sign to validate the prior study findings and to determine the therapeutic 
efficacy of recombinant factor VIIa or tranexamic acid. ${ }^{15-17} \mathrm{~A}$ more recent study using dynamic spot sign imaging with a biphasic CT perfusion protocol ${ }^{18}$ has confirmed 2 patterns of contrast extravasation associated with significantly different rates of leakage. These patterns, comprising a brisker active extravasation (spot sign) and slower postcontrast leakage (PCL), ${ }^{19}$ are also demonstrated with early and late structural imaging, ${ }^{10,19}$ dynamic CTA/CTP,${ }^{18}$ and biphasic or repeat delayed CTA acquisitions. ${ }^{12}$

Morphologic patterns and more recent studies illustrate that the spot sign is not an all-or-none phenomenon but constitutes a spectrum of extravasation. ${ }^{18,19}$ The extravasation rate likely significantly impacts timely and clinically meaningful hemostasis. ${ }^{20}$ A bleeding threshold likely exists beyond which prothrombotic treatment is futile, exposing patients to harmful adverse effects without hope of therapeutic benefit. ${ }^{21}$ Increasingly, new innovative surgical techniques are being developed to address contrast extravasation. ${ }^{22}$ Knowledge of the impact of the extravasation rate on therapeutic response is critical to stratify patients to the most appropriate therapies. An animal model of acute contrast extravasation in ICH could potentially inform the patient-selection process. We describe a novel MR imaging-integrated real-time swine model of acute hematoma growth and contrast extravasation.

\section{MATERIALS AND METHODS}

This study was conducted with the approval of the local Research Institute Animal Care Committee (Animal Use Protocol No. 12435) in compliance with the guidelines established by the Canadian Council on Animal Care and the Animals for Research Act. Fourteen 6- to 8-week-old (approximately 16-18 kg) Yorkshire male swine were chemically immobilized by using xylazine $\mathrm{HCl}$ $(2.2 \mathrm{mg} / \mathrm{kg})$, ketamine $(15 \mathrm{mg} / \mathrm{kg})$, and atropine $(0.05 \mathrm{mg} / \mathrm{kg})$ mixed together in a syringe by intramuscular injection into the dorsal neck or gluteal muscles. Anesthesia was initially maintained with $2 \%-3 \%$ isoflurane in oxygen $(>2 \mathrm{~L} / \mathrm{min})$. Percutaneous insertion of two $10-\mathrm{cm} 5 \mathrm{~F}$ catheters (Pinnacle; Terumo, Tokyo, Japan) via a Seldinger technique into the femoral artery and vein was performed for continuous arterial blood pressure monitoring and central line access, respectively. Physiologic monitoring at 5-minute intervals included clinical observation, tail pulse oximetry, rectal temperature, and blood pressure measurement through the femoral artery line. Fluid balance was maintained with intravenous normal saline $(10 \mathrm{~mL} / \mathrm{kg} / \mathrm{h})$ and equivalent normal saline volume replacement of any other losses.

The animal was transferred to the MR imaging suite and placed in a supine position with the head immersed in degassed water and its limbs secured (Fig 1). Degassed water served as a conduction medium for sonography emitted from an upwardly positioned modified clinical MR imaging-guided focused sonography brain system (ExAblate 4000; Insightec, Tirat Carmel, Israel). The focused sonography system consists of a 1024-element hemispheric phased array with a diameter of $30 \mathrm{~cm}$. Accurate anatomic localization of vessels within the basal ganglia was achieved by using MR imaging localization sequences. The basal ganglia represent a frequent site for ICH and are a commonly targeted location for experimental ICH models due to lower subarachnoid risk and intraventricular extension.

Following this procedure, $0.08-\mathrm{mL} / \mathrm{kg}(1.5 \mathrm{~mL})$ perflutren

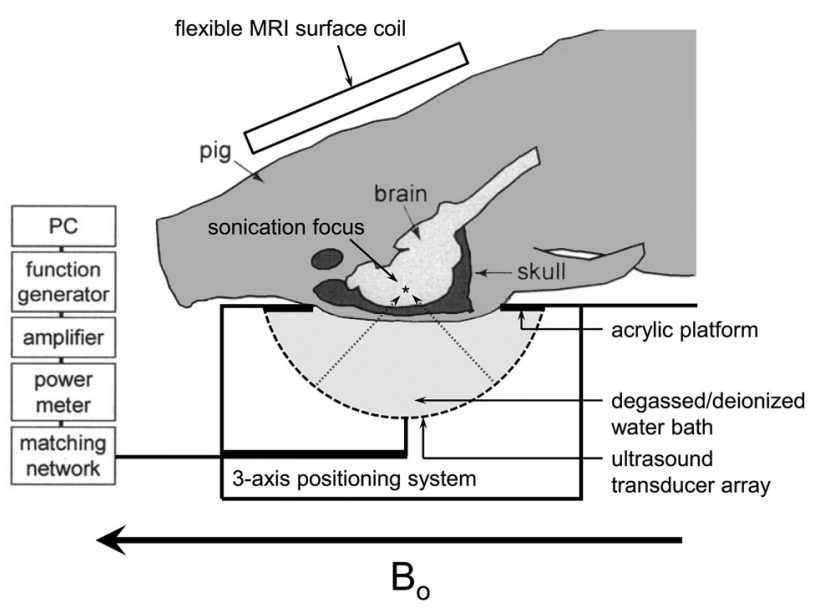

FIG 1. Schematic of swine model. Adapted with permission from McDannold N, King RL, Hynynen K. MRI monitoring of heating produced by ultrasound absorption in the skull: in vivo study in pigs. Magn Reson Med 2004;51:1061-65.

lipid microsphere infusion (Definity; Lantheus Medical Imaging, North Billerica, Massachusetts) and focused low-frequency sonography $(230 \mathrm{kHz})$ were delivered. MR imaging was performed before and after focused sonography vessel disruption by using 3T MR imaging (Discovery MR750; GE Healthcare, Milwaukee, Wisconsin) and a 6-channel phased array flexible coil (GE Healthcare). The following sequences were obtained at baseline: FSE T2 (TR/TE, 3000/71 ms; echo-train length, 4; FOV, $16 \times$ $16 \mathrm{~cm}$; slice thickness, $2 \mathrm{~mm}$; $256 \times 192)$; 3D spoiled gradient recalled-echo (GRE) T1 (TR/TE, 6/2 ms; FOV, $12 \times 12 \mathrm{~cm}$; slice thickness, $2 \mathrm{~mm}$ ); axial T2 FLAIR (TR/TE, 8000/127 ms; $12 \times 12$ $\mathrm{cm}$; slice thickness, $2 \mathrm{~mm}$; $128 \times 128)$; and fast GRE $(\mathrm{TR} / \mathrm{TE}$, $100 / 13 \mathrm{~ms} ; 12 \times 12 \mathrm{~cm}$; slice thickness, $2 \mathrm{~mm}$ ). Thirty seconds after perflutren injection and sonographic insonation, a dynamic contrast-enhanced sequence (DCE) (3D T1 spoiled GRE; TR/TE, $6 / 2 \mathrm{~ms} ; 12 \times 12 \mathrm{~cm}$; slice thickness, $3 \mathrm{~mm} ; 128 \times 128$; temporal sampling, 10 seconds; scan duration, 20 minutes) was performed. Gadobutrol, 1 mmol/mL (Gadovist; Bayer Schering Pharma, Toronto, Canada), was injected via a pump injector (Spectris MR injector; MedRad, Indianola, Pennsylvania; and Solaris; Bayer HealthCare, Pittsburgh, Pennsylvania) at $0.5 \mathrm{~mL} / \mathrm{kg}$ and $5 \mathrm{~mL} / \mathrm{s}$ followed by a $10-\mathrm{mL}$ normal saline bolus. A postgadolinium T1 study was performed at the termination of the DCE sequence (20 minutes after gadolinium injection). Fast GRE, FLAIR, and FSE T2 sequences were repeated following DCE and before procedure termination.

\section{Experimental Design}

Number of Experiments. Twenty-eight hemispheres were insonated; 16 hemispheres were used to determine optimal power delivery; and 12, to test the effect of burst length.

Power Delivery. On the basis of prior studies of blood-brain barrier disruption ${ }^{23-25}$ aiming at a temporary $\mathrm{BBB}$ opening but reporting complications of red blood cell extravasation, 2 power ranges were selected for evaluation. Sixteen hemispheres were subjected to basal ganglia insonation, with either 70-kW ( $n=8$ hemispheres) or $80-\mathrm{kW}$ ( $n=8$ hemispheres) power delivery at a 
constant burst length (10 ms, 1-Hz pulse-repetition frequency) and sonication duration (120 seconds). Animals were compared for DCE leakage rate, final hematoma size (see below), and vascular abnormality on histopathology. The 8 hemispheres at $80 \mathrm{~kW}$ were then used to compare with the 3 higher burst lengths also performed at $80 \mathrm{~kW}$.

\section{Burst Length}

The burst length determines the interaction time between sonography and bubbles and is central to the production of the desired cavitation effects, which result in blood vessel wall disruption. Altering burst length, therefore, determines the magnitude and threshold of disruption thereby modulating the rate of extravasation. ${ }^{26}$ Following $80-\mathrm{kW}$ power-level selection, 3 additional burst lengths ( $1000 \mathrm{~ms}$ [continuous wave], $800 \mathrm{~ms}$, and $600 \mathrm{~ms}$ ) in 4 hemispheres each were acquired. The 8 hemispheres at $10 \mathrm{~ms}$ by using the $80-\mathrm{kW}$ power delivery described above were included in a comparison of the 4 different burst lengths tested. Rate of extravasation and hematoma volumes were compared for each group. Within each burst-length subgroup, mean arterial pressure was increased at the time of insonation to achieve a $0 \%-60 \%$ elevation over baseline by using a $15-\mu \mathrm{g} / \mathrm{kg} / \mathrm{min}$ phenylephrine infusion. ${ }^{27}$

MR Image Processing. Hematoma and DCE contrast volume measurements were performed on GRE and DCE images, respectively, by using the auto-trace feature with Medical Image Processing, Analysis, and Visualization, Version 4.4.1, Center for Information Technology (National Institutes of Health, Bethesda, Maryland; http://mipav.cit.nih.gov). The largest region-of-interest volume within the temporal DCE dataset was selected as the final DCE volume. Rate of leakage (KPS) was measured within each region of contrast leakage from the permeability maps calculated using custom IDL software (Exelis Visual Information Solutions, Boulder, Colorado) by using a previously described unidirectional 2-compartment kinetic model. ${ }^{28}$ The DCE-derived region of interest, representing the volume of contrast leakage, was used for the permeability measurement from the KPS maps. Because the contrast leakage volumes were drawn on the same sequence from which the KPS maps were derived, the ROIs were intrinsically coregistered with the KPS maps.

Morphologic Characterization of Contrast Extravasation. On the basis of clinical studies, 2 distinct patterns of contrast leakage were identified. ${ }^{19}$ Active contrast extravasation demonstrated a brisk contrast leak with well-delineated margins, visible from early within the DCE sequence. Postcontrast leakage demonstrated slower leakage on DCE with ill-defined contrast enhancement becoming more confluent on a postcontrast T1 study performed following DCE (Figs 2 and 3).

Histopathologic Examination. Animals were observed for 2 hours following the last insonation and were euthanized by exsanguination followed by transcarotid perfusion of heparinized saline (2 L) and 4\% paraformaldehyde $(2 \mathrm{~L})$. The anterior and posterior cranium was removed to better facilitate immersionfixation in $4 \%$ paraformaldehyde. Following fixation, the brain was removed, blocked in 5-mm sections, and subjected to gross pathologic examination and photography. Regions of hematoma were sectioned at $5 \mu \mathrm{m}$ and stained with hematoxylin-eosin and

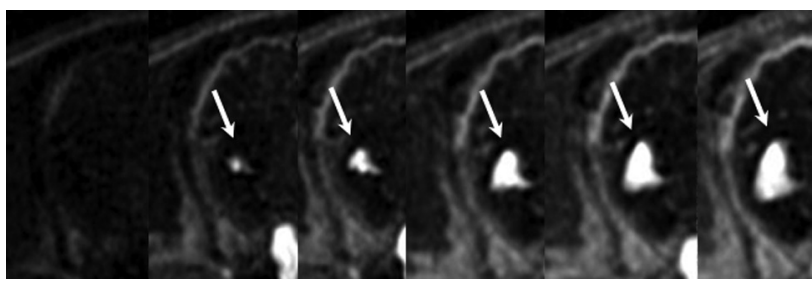

FIG 2. Individual frames from a DCE sequence demonstrating progressive accumulation of contrast with time within a right basal ganglia hematoma. Rapid accumulation of confluent and well-defined contrast consistent with active extravasation. KPS measured $6.39 \mathrm{~mL} /$ $\mathrm{min} / 100 \mathrm{~g}$.

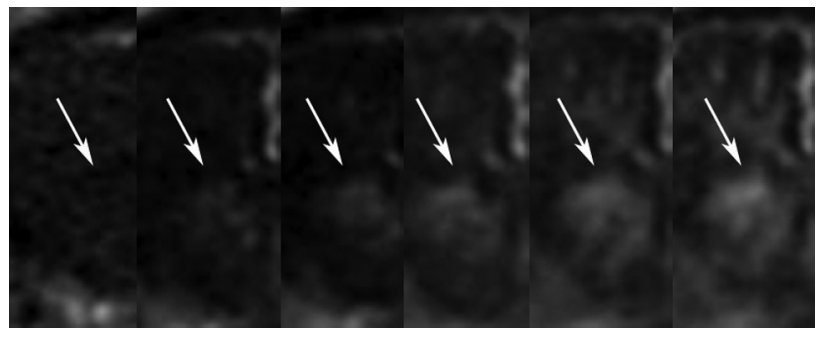

FIG 3. Individual frames from a DCE sequence demonstrating slow accumulation of contrast with time within a right basal ganglia hematoma consistent with postcontrast leakage. KPS measured $1.1 \mathrm{~mL}$ / $\mathrm{min} / 100 \mathrm{~g}$.

Masson elastin trichrome stain; and immunohistochemistry for smooth-muscle actin was used on selected sections.

\section{Statistical Analysis}

Statistical analysis was performed by using MedCalc (Version 12.7.0.0; MedCalc Software, Mariakerke, Belgium). Median (interquartile range [IQR]) contrast leakage, DCE contrast, and GRE hematoma volumes were calculated and compared between 70 and $80-\mathrm{kW}$ power groups and between each burst-length category by using the Mann-Whitney $U$ test. Spearman correlation coefficients were calculated for increasing burst length and GRE hematoma and DCE volumes. Correlation among the rate of contrast leak, GRE hematoma volume, and mean arterial pressure ratio was assessed for each burst-length group. The association between burst length and morphologic pattern of contrast leakage (CE versus PCL) was tested with the Fisher exact test. We compared the rate of leakage, hematoma, and DCE volumes between $\mathrm{CE}$ and PCL using the Mann-Whitney $U$ test. $P<.05$ was considered significant.

\section{RESULTS}

\section{Power Determination}

Median rates of contrast leakage were similar at $70 \mathrm{~kW}$ and $80 \mathrm{~kW}$ (4.5 mL/min/100 g [IQR, 2.4-25.1] versus $3.2 \mathrm{~mL} / \mathrm{min} / 100 \mathrm{~g}$ [IQR, 1.2-7.6]; $P=.75$, respectively), though the range of values was more heterogeneous in the $70-\mathrm{kW}$ group. Similar median (IQR) GRE hematoma $\left(0.6 \mathrm{~cm}^{3}[0.4-1.5]\right.$ versus $0.5 \mathrm{~cm}^{3}[0.4-$ $0.8] ; P=.98)$ and DCE volumes $\left(0.1 \mathrm{~cm}^{3}[0.1-0.7]\right.$ versus $0.2 \mathrm{~cm}^{3}$ $[0.1-0.4] ; P=.39)$ were present. Due to the more homogeneous values achieved at $80 \mathrm{~kW}$, this power level was selected for further study. Histopathologic analysis of cases performed at $10 \mathrm{~ms}$ demonstrated predominantly focal injury of small intracerebral blood vessel walls $(40-70 \mu \mathrm{m})$ characterized by segmental bland, gran- 


\begin{tabular}{lccccc}
\multicolumn{6}{c}{ Comparison of contrast leakage rate, GRE hematoma, and DCE volume by burst-length subgroup for $\mathbf{2 0}$ hemispheres at $\mathbf{8 0}$ kW power } \\
\hline $\begin{array}{l}\text { Burst Length }(\mathbf{m s}) \\
(\boldsymbol{n}=\text { Hemispheres })\end{array}$ & $\begin{array}{c}\text { Contrast Leak Median (IQR) } \\
(\mathbf{m L} / \mathbf{m i n} / 100 \mathbf{~})\end{array}$ & $\begin{array}{c}\text { GRE Hematoma } \\
\text { Volume }\left(\mathbf{c m}^{\mathbf{3}}\right)\end{array}$ & $\begin{array}{c}\text { DCE Contrast } \\
\text { Volume }\left(\mathbf{c m}^{3}\right)\end{array}$ & $\begin{array}{c}\boldsymbol{\rho} \text { KPS-to-Hematoma } \\
\text { Volume }\end{array}$ & $\begin{array}{c}\boldsymbol{\rho} \text { KPS-to-MAP } \\
\text { Ratio }\end{array}$ \\
\hline $1000(n=4)$ & $17.4(9.5-29.0)$ & $1.6(1.2-2.1)$ & $1.3(1.1-1.4)$ & $0.8(-0.7-0.9, P=.3)$ & $0.8(-0.6-0.9, P=.2)$ \\
$800(n=4)$ & $6.0(4.2-7.4)$ & $0.9(0.7-1.7)$ & $0.4(0.2-0.7)$ & $0.7(-0.5-0.9, P=.2)$ & $0.5(-0.7-0.9, P=.7)$ \\
$600(n=4)$ & $2.9(1.5-3.7)$ & $1.0(0.4-1.6)$ & $0.4(0.2-1.0)$ & $0.6(-0.8-1.0, P=.4)$ & $0.2(-0.9-0.9, P=.8)$ \\
$10(n=8)$ & $3.21(1.7-7.3)$ & $0.5(0.4-0.9)$ & $0.2(0.1-0.4)$ & $0.3(-0.4-0.7, P=.5)$ & $0.6(-0.1-0.9, P=.01)$ \\
\hline
\end{tabular}

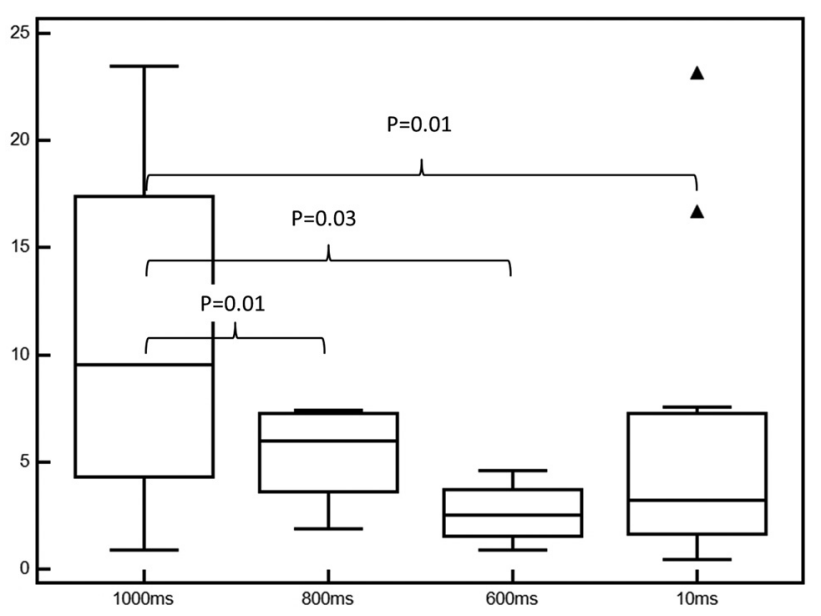

FIG 4. Rate of contrast leakage stratified by burst length. Median, $\mathrm{IQR}$, and $95 \% \mathrm{Cl}$ values are displayed as bar, box, and whiskers respectively.

ular smooth-muscle eosinophilia, shrinkage, and hyperchromatism of their nuclei occasionally associated with margination of neutrophils. Larger vessels $(70-120 \mu \mathrm{m})$ were rarely affected and showed relatively minor abnormality. The order of magnitude of small-vessel involvement was not unexpected, given that the initially selected parameters mirrored those used to disrupt the blood-brain barrier. ${ }^{23-25}$ Increasing the burst length reduces the amount of time for bubbles to enter the microcirculation before cavitating, theoretically shifting this process to larger sized vessels.

\section{Optimal Burst-Length Determination}

Median (IQR) rates of contrast leak, DCE contrast and hematoma volume by burst length and correlation for contrast leak mean arterial pressure elevation (MAP) ratio, and DCE contrast and GRE hematoma volume are listed in the Table. Median rates of contrast leakage were significantly higher at $1000 \mathrm{~ms}$ compared with $800-\mathrm{ms}(P=.01), 600-\mathrm{ms}(P=.02)$, and $10-\mathrm{ms}(P=.01)$ burst lengths (Table and Fig 4$)$. Increasing burst length was associated with increasing DCE contrast ( $\rho=0.55$; 95\% CI, $0.11-$ $0.81 ; P=.02$ ) and hematoma volumes ( $\rho=0.54$; 95\% CI, $0.17-$ $0.78 ; P=.007)$. Within each burst-length subgroup, incremental contrast leakage was seen with an increasing MAP ratio ( $\rho=$ 0.2-0.8; Table). The strongest correlation between KPS and hematoma volume and KPS and MAP ratio was present in the 1000-ms group. The 800- and 10-ms subgroups demonstrated moderate KPS-to-MAP correlation. KPS-to-GRE hematoma-volume correlation was moderate in the 600- and 800-ms subgroups.

\section{Morphologic Patterns of Leakage in 20 Hemispheres at $80 \mathrm{~kW}$}

The median (IQR) rate of contrast leakage (CE or PCL) for all experiments was $5.2(2.9-10.7) \mathrm{mL} / \mathrm{min} / 100 \mathrm{~g}$. CE was more common at higher (1000 and 800) than lower (600 and 10) burst lengths ( $P=$ .03). Median (IQR) CE KPS was significantly elevated compared with PCL (11.3 mL/min/100 g; 6.3-23.2 versus $2.4 \mathrm{~mL} / \mathrm{min} / 100 \mathrm{~g}$; $1.1-3.1 ; P<.001)$. The median (IQR) final GRE hematoma volume was significantly higher for CE compared with PCL $\left(0.7 \mathrm{~cm}^{3} ; 0.4-1.6\right.$ versus $\left.0.4 \mathrm{~cm}^{3} ; 0.06-0.7 ; P=.02\right)$. Similarly, CE demonstrated a median (IQR) DCE contrast volume of $0.4 \mathrm{~cm}^{3}(0.1-1.3)$ compared with PCL $\left(0.04 \mathrm{~cm}^{3} ; 0.02-0.1 ; P=.01\right)$.

\section{Histologic Examination of the Brain}

Multiple whole-mount hematoxylin-eosin coronal step sections through the intracerebral hemorrhage were screened microscopically. Although the extent of bleeding and the vascular changes varied with the sonication parameters, a distinctive pattern of vascular injury emerged within arteries and arterioles typically 50-100 $\mu \mathrm{m}$ in diameter. Pathologic vessels were distributed throughout the sonicated tissue and in the subarachnoid space, associated with varying degrees of subarachnoid bleeding, particularly in the interhemispheric fissure. Sonication changes, which we have termed "sonication vasculopathy," were characterized by fairly extensive vascular injury, demonstrating varying degrees of segmental blood vessel wall bland eosinophilia and loss of nuclear detail. In some instances, early neutrophil margination was present. Abnormality extended for some distance along the blood vessels and, in some instances, alternated with more normal vascular structure, producing segmental lesions. Associated with these changes were red cells in the blood vessel wall suggesting "mini dissection." Almost without exception, the injured vessels were associated with perivascular collections of red blood cells of various sizes ranging from petechiae to substantial intracerebral hematomas. Many of the involved small vessels showed focal defects blending with perivascular fibrin-platelet debris, both in the small petechial hemorrhages and around the larger hematomas. Increasing burst length was characterized by involvement of larger perforating vessels in which bland mural eosinophilia, reminiscent of necrosis, and rupture sites were identified (Fig 5A). Smaller vessels were less involved in cases with large hematomas, and greater difficulty was experienced identifying a ruptured vessel in larger hematomas. Although this could be a problem of sampling, the presence of actin-immunopositive fragments supports extensive segmental vessel injury and fragmentation.

\section{DISCUSSION}

We report the first MR imaging-integrated real-time swine model of contrast extravasation and hematoma growth. The median rate of contrast leakage reported is consistent with that reported by d'Esterre et $\mathrm{al}^{18}$ for humans, showing a leakage rate of $6.5 \pm 1.6$ $\mathrm{mL} / \mathrm{min} / 100 \mathrm{~g}$. The 1000 -ms subgroup emerged as the optimal model for further study of prothrombotic agents by virtue of a strong correlation between the contrast leakage rate and final he- 

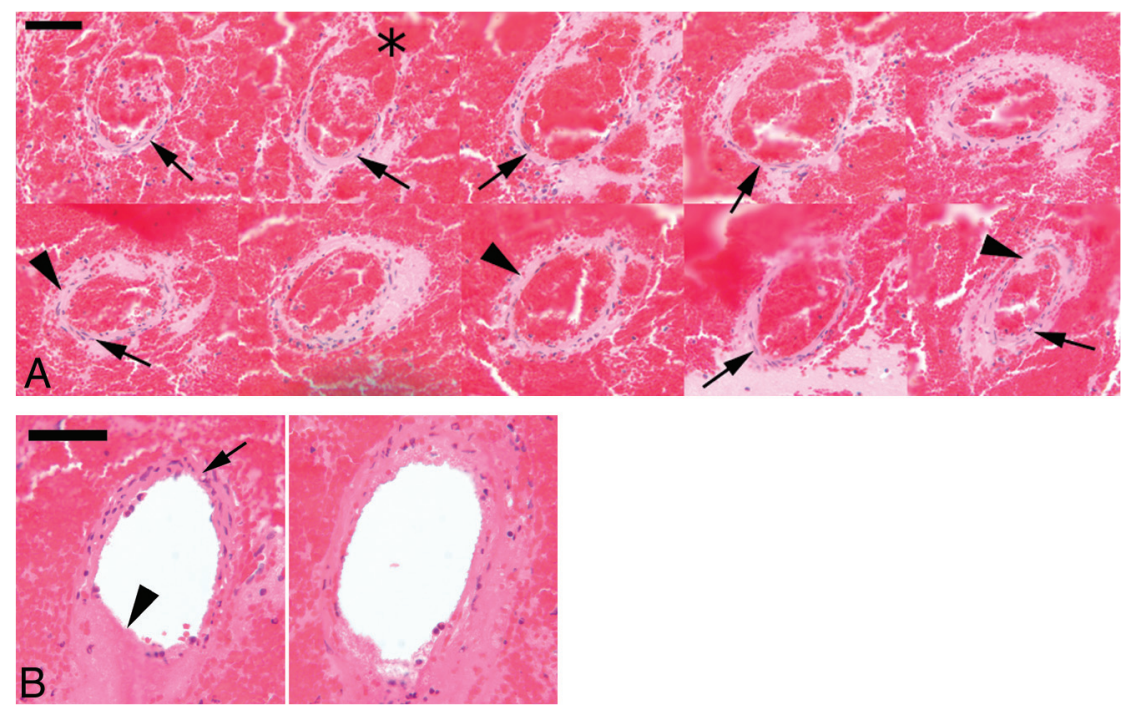

FIG 5. A, Hematoxylin-eosin stain showing multiple levels along the course of a small intracerebral artery, encased in a recent hematoma. The rupture site is illustrated in most of the top row profiles (asterisk). Although fragments of normal vessel wall remain (arrows), there are many areas, particularly in the lower row, where the apparently intact vessel wall is formed by bland eosinophilic material whose appearance is reminiscent of necrosis (arrowheads). B, Penetrating branch of the circle of Willis. The profile on the left is close to the inferior/basilar surface, whereas the one on the right is deeper in the brain. A rupture site in the superficial profile is indicated by an arrowhead. Note the intact segment of the artery (arrow). A small rupture site in the deeper vessel portion is similarly present, but most of the vessel wall exhibits loss of the normal microscopic anatomy and replacement by bland eosinophilia. Scale bar $=50 \mu \mathrm{m}$.

matoma size. The strong correlation between the MAP ratio and leakage rate within the 1000-ms subgroup indicates an ability to further regulate the leakage rate through blood pressure manipulation.

Although contrast extravasation is best characterized in the CT literature, Murai et $\mathrm{al}^{29}$ described MR imaging contrast extravasation as a marker of ongoing hemorrhage in acute ICH. Of 108 patients with acute hypertensive $\mathrm{ICH}$, contrast extravasation was seen in 39 MR imaging and 17 cerebral angiography studies. Significant correlation with follow-up hematoma growth was confirmed. A recent case report describes a patient experiencing an acute ICH while undergoing MR imaging for unrelated reasons, illustrating progressive contrast extravasation and hematoma formation. ${ }^{4}$ The volume of contrast extravasation in the present model was smaller than the final GRE hematoma size in all burst-length groups. Notably, this difference was most pronounced at lower burst lengths. If the difference was attributable to gradient blooming, similar disparity should be present in each group. It is more likely that the greater difference at lower burst length reflects the notion that not all contrast opacification represents irreversible blood-brain barrier disruption and extravasation. ${ }^{30}$ The greater correlation at higher burst lengths between the rate of contrast leak and hematoma size underscores the association between the increased sonography intensity exposure and the increased severity of vessel injury, also confirmed by histopathologic analysis and prior studies. ${ }^{26}$

The present technique represents a significant evolution over existing ICH models by mimicking mural disruption suspected in human ICH pathophysiology. In humans, the vascular abnormalities underlying ICH formation and expansion are poorly understood. Pathologic examination of ICH specimens is hindered by the friable nature of formalin-fixed blood and the time-consuming and technically demanding processes that are needed to conduct a "complete" histopathologic survey of large hematomas. Observations derived from pathologic studies by Fisher ${ }^{3}$ report focal rupture of a $0.6-\mathrm{mm}$ arteriole with aneurysmal dilation in hypertensive ICH, fibrin globes, ${ }^{7}$ and microdissections. ${ }^{31}$ Common to the many proposed etiologies in the diseased state is the segmental involvement of vessels with "skip lesions." ${ }^{3}$ Vessel wall rupture in the present model is induced by intraluminal cavitation mediated through the interaction of sonography waves and injected bubbles. Our histopathologic analysis revealed a mini-dissection appearance and also frank arterial disruption with concomitant red blood cell extravasation mirroring a recent histopathologic description of dissection in a $920-\mu \mathrm{m}$ artery found at the periphery of a spot-positive hematoma. $^{32}$

The proposed swine model serves as a paradigm for contrast extravasation in human ICH, offering a unique opportunity to study the efficacy of drug and noninvasive imaging-guided therapies. The use of contrast extravasation to select patients most likely to benefit from therapy has been recently incorporated into the American Heart Association guidelines. ${ }^{33}$ Swine are the National Institute of Neurological Disorders and Stroke model of choice for ICH study because they offer several advantages over traditional animal models, including proportional gray and white matter representation similar to that in humans and the ability to create hematoma volumes 20-30 times larger than those in rodent species. ${ }^{34,35}$ The MR imaging integration environment allows real-time monitoring and objective measurement of hematoma formation and contrast extravasation with susceptibility and dynamic contrast-enhanced $\mathrm{T} 1$ sequences, respectively. Unlike rodents, swine do not have potentially toxic hemostatic doses of recombinant factor VIIa, ${ }^{36}$ a critical limitation given the use of the drug in current hemostatic ICH studies (Spot Sign for Predicting and Treating ICH Growth, "Spot Sign" Selection of Intracerebral Hemorrhage to Guide Hemostatic Therapy). ${ }^{15,16}$

Study limitations include the small sample size because of the need to test multiple burst lengths. MR imaging was selected due to the integration of sonography, facilitating rapid scan acquisition following insonation. A CT model is feasible and faster than MR imaging but would require removal of the sonication device before scanning, thereby increasing the delay between vessel disruption and imaging, complicating coregistration, and requiring repeat calibration if further insonation is considered. Other disadvantages of the present model include the significantly higher experimental cost compared with rodent models. The first iteration of the model was complicated by subarachnoid hemorrhage formation due to sonography wave refraction caused by the flat swine skull. Future model iterations are underway that will miti- 
gate or minimize these effects. The model application is, therefore, presently limited to the study of acute ICH formation and contrast extravasation.

\section{CONCLUSIONS}

We describe a swine model of ICH formation and contrast extravasation. We anticipate that the model will facilitate the study of therapeutic interventions against active contrast extravasation and its effect on hematoma formation and clinical outcome.

\section{ACKNOWLEDGMENTS}

We thank the animal technologists and Comparative Research and Sunnybrook Research Institute Animal Care Committee without whom this work would not be possible.

Disclosures: Richard I. Aviv—RELATED: Grant: Heart and Stroke Foundation (grant No.7299).* Thien Huynh—RELATED: Grant: Physician Services Incorporated, * Canadian Institute for Health Research.* Dan Dumont-UNRELATED: Grants/Grants Pending: Heart and Stroke Foundation, Comments: The Heart and Stroke Foundation paid for research on Vasculotide, but the topic was completely unrelated to this current manuscript, Patents (planned, pending or issued): Vasomune, Comments: Vasculotide is licensed to Vasomune and forms the basis for this startup. There are currently 3 active patents being pursued, Other: I am a codiscoverer of Vasculotide, and I act as a chair of the Scientific advisory board for Vasomune Therapeutics. *Money paid to the institution.

\section{REFERENCES}

1. Broderick JP, Adams HP Jr, Barsan W, et al. Guidelines for the management of spontaneous intracerebral hemorrhage: a statement for healthcare professionals from a special writing group of the Stroke Council, American Heart Association. Stroke 1999;30:905-15

2. Broderick J, Connolly S, Feldmann E, et al. Guidelines for the management of spontaneous intracerebral hemorrhage in adults: 2007 update: a guideline from the American Heart Association/American Stroke Association Stroke Council, High Blood Pressure Research Council, and the Quality of Care and Outcomes in Research Interdisciplinary Working Group. Stroke 2007;38:2001-23

3. Fisher CM. Hypertensive cerebral hemorrhage: demonstration of the source of bleeding. J Neuropathol Exp Neurol 2003;62:104-07

4. Jeong D, Jhaveri MD, Prabhakaran S. Magnetic resonance imaging characteristics at onset of spontaneous intracerebral hemorrhage. Arch Neurol 2011;68:826-27

5. Bermejo PG, Garcia JA, Perez-Fernandez S, et al. Spot sign and liveimaged dramatic intracerebral hematoma expansion. Neurology 2010;75:834

6. Rizos T, Rohde S, Dorner N, et al. Ongoing intracerebral bleeding despite hemostatic treatment associated with a spot sign in a patient on oral anticoagulation therapy. Cerebrovasc Dis 2009;28:623-24

7. Fisher CM. Pathological observations in hypertensive cerebral hemorrhage. J Neuropathol Exp Neurol 1971;30:536-50

8. Brott T, Broderick J, Kothari R, et al. Early hemorrhage growth in patients with intracerebral hemorrhage. Stroke 1997;28:1-5

9. Goldstein JN, Fazen LE, Snider R, et al. Contrast extravasation on CT angiography predicts hematoma expansion in intracerebral hemorrhage. Neurology 2007;68:889-94

10. Wada R, Aviv RI, Fox AJ, et al. CT angiography "spot sign” predicts hematoma expansion in acute intracerebral hemorrhage. Stroke 2007;38:1257-62

11. Kim J, Smith A, Hemphill JC 3rd, et al. Contrast extravasation on CT predicts mortality in primary intracerebral hemorrhage. AJNR Am J Neuroradiol 2008;29:520-25

12. Delgado Almandoz JE, Yoo AJ, Stone MJ, et al. Systematic characterization of the computed tomography angiography spot sign in primary intracerebral hemorrhage identifies patients at highest risk for hematoma expansion: the spot sign score. Stroke 2009;40: 2994-3000
13. Demchuk AM, Dowlatshahi D, Rodriguez-Luna D, et al. Prediction of haematoma growth and outcome in patients with intracerebral haemorrhage using the CT-angiography spot sign (PREDICT): a prospective observational study. Lancet Neurol 2012;11:307-14

14. Li N, Wang Y, Wang W, et al. Contrast extravasation on computed tomography angiography predicts clinical outcome in primary intracerebral hemorrhage: a prospective study of 139 cases. Stroke 2011;42:3441-46

15. Clinicaltrials.gov. The Spot Sign for Predicting and Treating ICH Growth Study (STOP-IT). Published 15 December 2008. Updated 12 June 2012. http://wwwclinicaltrialsgov/ct2/show/NCT00810888? term =stop-it\&rank=1. Accessed February 20, 2013

16. Clinicaltrials.gov. "Spot Sign” Selection of Intracerebral Hemorrhage to Guide Hemostatic Therapy (SPOTLIGHT). Published 20 May 2011. Updated 12 October 2012. http://wwwclinicaltrialsgov/ct2/ show/NCT01359202?term $=$ spotlight\&rank=1. Accessed February 20, 2013

17. Clinicaltrials.gov. STOP-AUST: The Spot Sign and Tranexamic Acid On Preventing ICH Growth-AUStralasia Trial. Published 3 October 2012. Updated 14 December 2012. http://wwwclinicaltrialsgov/ct2/ show/NCT01702636?term $=$ stop-aust\&rank $=1$. Accessed January 20, 2013

18. d'Esterre CD, Chia TL, Jairath A, et al. Early rate of contrast extravasation in patients with intracerebral hemorrhage. AJNR Am JNeuroradiol 2011;32:1879-84

19. Ederies A, Demchuk A, Chia T, et al. Postcontrast CT extravasation is associated with hematoma expansion in CTA spot negative patients. Stroke 2009;40:1672-76

20. Sapsford W, Watts S, Cooper G, et al. Recombinant activated factor VII increases survival time in a model of incompressible arterial hemorrhage in the anesthetized pig. J Trauma 2007;62:868-79

21. Diringer MN, Skolnick BE, Mayer SA, et al. Thromboembolic events with recombinant activated factor VII in spontaneous intracerebral hemorrhage: results from the Factor Seven for Acute Hemorrhagic Stroke (FAST) trial. Stroke 2010;41:48-53

22. Nagasaka T, Inao S, Wakabayashi T. What does the CT angiography "spot sign" of intracerebral hemorrhage mean in modern neurosurgical settings with minimally invasive endoscopic techniques? Neurosurg Rev 2013;36:341-48

23. Hynynen K, McDannold N, Vykhodtseva N, et al. Noninvasive MR imaging-guided focal opening of the blood-brain barrier in rabbits. Radiology 2001;220:640-46

24. Hynynen K, McDannold N, Sheikov NA, et al. Local and reversible blood-brain barrier disruption by noninvasive focused ultrasound at frequencies suitable for trans-skull sonications. Neuroimage 2005;24:12-20

25. Hynynen K, McDannold N, Vykhodtseva N, et al. Focal disruption of the blood-brain barrier due to $260-\mathrm{kHz}$ ultrasound bursts: a method for molecular imaging and targeted drug delivery. J Neurosurg 2006;105:445-54

26. McDannold N, Vykhodtseva N, Hynynen K. Effects of acoustic parameters and ultrasound contrast agent dose on focused-ultrasound induced blood-brain barrier disruption. Ultrasound Med Biol 2008;34:930-37

27. Troncy E, Francoeur M, Salazkin I, et al. Extra-pulmonary effects of inhaled nitric oxide in swine with and without phenylephrine. $\mathrm{Br} J$ Anaesth 1997;79:631-40

28. Roberts HC, Roberts TP, Bollen AW, et al. Correlation of microvascular permeability derived from dynamic contrast-enhanced MR imaging with histologic grade and tumor labeling index: a study in human brain tumors. Acad Radiol 2001;8:384-91

29. Murai Y, Ikeda Y, Teramoto A, et al. Magnetic resonance imagingdocumented extravasation as an indicator of acute hypertensive intracerebral hemorrhage. J Neurosurg 1998;88:650-55

30. Molina CA, Alvarez-Sabin J, Montaner J, et al. Thrombolysis-related hemorrhagic infarction: a marker of early reperfusion, reduced infarct size, and improved outcome in patients with proximal middle cerebral artery occlusion. Stroke 2002;33:1551-56 
31. Challa VR, Moody DM, Bell MA. The Charcot-Bouchard aneurysm controversy: impact of a new histologic technique. J Neuropathol Exp Neurol 1992;51:264-71

32. Huynh TJ, Keith J, Aviv RI. Histopathological characteristics of the "spot sign" in spontaneous intracerebral hemorrhage. Arch Neurol 2012;69:1654-55

33. Morgenstern LB, Hemphill JC 3rd, et al. Guidelines for the management of spontaneous intracerebral hemorrhage: a guideline for healthcare professionals from the American Heart Association/ American Stroke Association. Stroke 2010;41:2108-29
34. NINDS ICH Workshop Participants. Priorities for clinical research in intracerebral hemorrhage: report from a National Institute of Neurological Disorders and Stroke workshop. Stroke 2005;36: e23-41

35. Wagner KR. Lobar intracerebral hemorrhage model in pigs: rapid edema development in perihematomal white matter. Stroke 1996;27:490-97

36. Zhang J, Groff RF 4th, Chen XH, et al. Hemostatic and neuroprotective effects of human recombinant activated factor VII therapy after traumatic brain injury in pigs. Exp Neurol 2008;210:645-55 